\title{
Screening for Mild Cognitive Impairment through Digital Biomarkers of Cognitive Performance in Games
}

\section{Karsten Gielis}

$\mathrm{KU}$ Leuven

Andreas Vesaliusstraat 13,

3000 Leuven

karsten.gielis@kuleuven.be
Permission to make digital or hard copies of part or all of this work for personal or classroom use is granted without fee provided that copies are not made or distributed for profit or commercia advantage and that not made or this notice and the full citation on the first page. Copyric for third-party components of this work must be honored. For all other

CHI PLAY EA '19, October 22-25, 2019, Barcelona, Spain

(c) 2019 Copyright is held by the owner/author(s).

ACM ISBN 978-1-4503-6871-1/19/10.

https://doi.org/10.1145/3341215.3356332

\section{Abstract}

This extended abstract describes the ongoing "Dr. Solitaire" project, which explores the possibilities of commercial off-the-shelf games to detect mild cognitive impairment by means of digital biomarkers of cognitive performance. A toolkit using computer vision and image processing was built for the standard Microsoft Solitaire Collection to capture these digital biomarkers at realtime. Preliminary studies conducted to detect cognitive decline due to cognitive ageing imply that cognitive decline can be detected through card gameplay and that machine learning algorithms can be trained to detect the intricacies of cognitive decline.

\section{Author Keywords}

Digital Biomarkers; Card Games; Machine Learning; Mild Cognitive Impairment;

\section{CSS Concepts}

- Human-centered computing Human computer interaction (HCI) - Applied computing Life and

medical sciences • Computing

methodologies Supervised learning by classification

\section{Introduction}

Around the world, every three seconds a new case of dementia arises. In 2018, 50 million people were living with dementia. This number is projected to reach 82 million by 2030 and 152 million by 2050 . The total 
estimated worldwide cost, not including the emotional toll taken on friends and family, is estimated to be one trillion US dollars in 2018 and two trillion US dollars by 2030[36].

Next to this influx of new cases, one of the greatest challenges of dementia is detecting the illness in early stages, where irreversible neuronal damage is still limited. The precursor stage of dementia is referred to as mild cognitive impairment (MCI) and can be defined as "the borderland between the cognitive changes of aging and very early dementia"[29]. Detecting dementia in this early stage is crucial; to mitigate disease progression, to make decisions, to follow-up and ensure necessary support for relatives, and to timely start cognitive therapy or medicinal treatments (e.g. donepezil) $[8,22,24,28,30]$. These steps are all crucial to prolong and improve quality of life. Despite the importance of early detection, studies report that $35 \%$ to $90 \%$ of dementia cases are overlooked and that there is a severe underdetection of cognitive impairments $[4,9,32]$

There are four prominent challenges of conventional neuropsychological assessments that need to be overcome to tackle these issues[13]. First, assessments are obtrusive. They require the frail older adult to spend time and resources to go to the memory clinic, breaking their regular routine. To be better equipped for challenges of the future, new neuropsychological tools should be less obtrusive. Second, assessments have low ecological validity. The tasks performed during neuropsychological assessment are different from everyday behavior, require discrete responses to single events, and are conducted in controlled environments [14]. To improve ecological validity, such a tool should be incorporated in daily life, with serial or even parallel responses to a stream of tasks [14]. Third, they are episodic, only providing momentary snapshots of the patients' cognitive status. These infrequent single-point-in-time measurements make the results vulnerable to unwanted factors such as tiredness, stress, or dehydration $[1,2,7]$. Ideally, assessment should happen on a frequent basis (i.e. weekly or even daily), increasing the temporal and spatial resolution of data [15]. These repeated measures could lead to a more clear personal cognitive profile, as opposed to comparing results to standard scores, sensitive to small deviations in cognitive performance[27]. Finally, assessments are labor intensive and thus poorly scalable. Thorough neuropsychological assessment requires resources such as space, staff, and equipment. Looking at the fact that most new cases of dementia will arise in emerging countries, where resources are more limited (Figure 1),

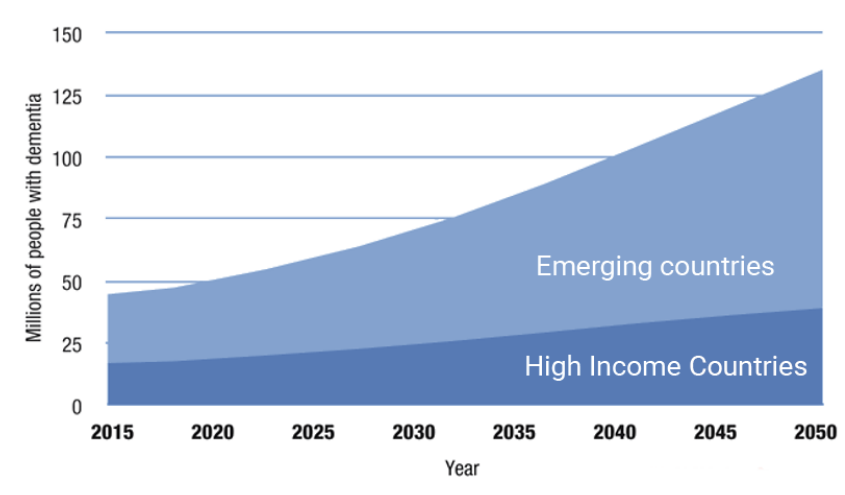

Figure 1. Number of dementia cases in emerging countries compared to high income countries, adapted from [22]. 
this scalability issue will become more and more imperative in the future[35].

These impending issues have spurred calls across multiple instances. In 2017, the World Health Organization endorsed the Global action plan on the public health response to dementia, focusing amongst others on diagnosing cognitive impairments in an earlier stage[34]. More recently, in June 2019, the Canadian government has published its first national strategy on dementia, freeing up to 70 million dollars for initiatives in the field[37], with one of the areas of focus specifically directed at enabling early diagnosis.

As a response to these calls, impending challenges, and the need for an extra tool for neuropsychological assessment, this research project was devised. It explores a novel method to detect and monitor cognitive impairments by capturing cognitive information through causal gameplay. More specifically, we capture this cognitive information in the form of digital biomarkers of cognitive performance found in the card game Klondike Solitaire [25].

\section{Research Objective}

The main objective of the Dr. Solitaire project is to explore whether Klondike Solitaire can be used to detect MCI. To this end, we focus on answering the following questions: Which cognitive functions are primarily addressed while playing Klondike Solitaire? Which digital biomarkers, found in Klondike Solitaire, are most indicative of cognitive performance? And finally, is it possible to detect cognitive fluctuations [23], the phenomenon of temporary alterations in cognition, attention, and arousal via play of Solitaire?

\section{Meaningful Play for Meaningful Results}

Detecting cognitive status by means of games is not a novel concept; for years researchers have explored the possibilities of games as medium to extract cognitive information[5]. Games provide rules and playful worlds which push players' to exert effort [12]. They are a natural source of information on behavior, cognitive performance, motor performance, social behavior and affect [25]. This has inspired exemplar games such as Space Fortress and Sea Hero Quest [11][26].

Unfortunately, these serious games ("Games with a serious goal, rather than entertainment, enjoyment or fun, as their primary purpose" [16]) face other issues. Their funding is often limited when compared to AAA developers. As such it is likely that the game will be outdated by the time the game is programmed, funding is gathered, and medical ethical clearance is approved [10]. In addition, the gameplay can be a frustrating experience, as they are developed by psychologists and not game developers[5]. As a consequence, such games can suffer in engagement, especially in longitudinal studies[6].

That is why recently, research has pivoted towards detecting cognitive decline using meaningful play (i.e. commercial off-the-shelf games that are part of the social fabric of the gamer's life) $[3,20,21,31,33]$.

\section{Research Approach}

Our research approach is be divided into four main components.

1. An expert study to define the most prominent cognitive functions addressed in Klondike Solitaire. 
2. A composition of digital biomarkers of cognitive performance and building of tools to capture said digital biomarkers.

3. A study to define the characteristics of MCI in gameplay, using machine learning models to discern older adults with MCI from their healthy counterparts.

4. A longitudinal study to further investigate the impact of MCI on game performance, focusing on detecting cognitive fluctuations and the creation of individual cognitive profiles.

\section{Results}

A preliminary work-in-progress study, where 22 player actions of Klondike Solitaire were mapped to 10 cognitive functions, by experts in neuropsychology and $\mathrm{MCY}$, indicated that the most prominent cognitive functions addressed while playing Klondike Solitaire were attention, executive function, object recognition, abstraction and memory. In addition, the experts remarked that such a tool would be valuable for its possibility to screen cognitive impairments

longitudinally, in a non-intrusive way, whilst being less susceptible to practice effects[17].

To investigate the possibilities of card games to measure cognitive performance, A toolkit was built using computer vision and image processing to analyze card gameplay from the standard Microsoft Solitaire Collection. Results showed that this technique allows for unobtrusively measuring digital biomarkers of cognitive performance at real-time[18].

Using this toolkit, a first exploratory study was done to assess cognitive decline due to cognitive aging. Digital biomarkers of cognitive performance were collected from three distinctive age groups (18-25,40-55,65+) with 52 participants, playing for a total of 130 game rounds. We found features retained for the model to support theories on fluid intelligence and cognitive functions sensitive to cognitive aging. When training a machine learning model to discern age groups, performance metrics suggest that our model is successful in classifying young and older participants. However, classification of middle-aged players showed to be problematic [19].

\section{Future work}

These prior studies were critical in validating Klondike Solitaire as game of choice, testing our toolkit, and piloting the capabilities of machine learning to assess cognitive decline. The results strengthen us to further pursue the possibilities of digital biomarkers in digital card games to detect MCI.

First, a study is planned with older adults with MCI and healthy older adults. Cognitive test batteries will be taken from every participant to be able to correlate cognitive functions to specific gameplay behavior. In addition, machine learning models will be trained to test the viability of screening for MCI.

Finally, a longitudinal study is planned where older adults with MCI and healthy older adults will play the game for a longer period of time. In this study we verify whether cognitive fluctuations can be detected and see where both groups differ in cognitive profile. Additionally, we will investigate whether retention rates are better when playing Solitaire than when playing a serious game for the assessment of cognitive functioning. 


\section{References}

1. Ana Adan. 2012. Cognitive performance and dehydration. Journal of the American College of Nutrition 31, 2: 71-78.

2. Paula Alhola and Päivi Polo-Kantola. 2007. Sleep deprivation: Impact on cognitive performance.

Neuropsychiatric Disease and Treatment 3, 5: 553567.

3. Pauline L. Baniqued, Hyunkyu Lee, Michelle W. Voss, et al. 2013. Selling points: What cognitive abilities are tapped by casual video games? Acta Psychologica 142, 1: 74-86.

4. Emma Bartfay, Wally J. Bartfay, and Kevin M. Gorey. 2013. Prevalence and correlates of potentially undetected dementia among residents of institutional care facilities in Ontario, Canada, 2009-2011: Prevalence of potentially undetected dementia. International Journal of Geriatric Psychiatry 28, 10: 1086-1094.

5. Walter R. Boot. 2015. Video games as tools to achieve insight into cognitive processes. Frontiers in Psychology 6.

6. Walter R. Boot, Arthur F. Kramer, Daniel J. Simons, Monica Fabiani, and Gabriele Gratton. 2008. The effects of video game playing on attention, memory, and executive control. Acta psychologica 129, 3: 387-398. 7. Lyle E. Bourne Jr and Rita A. Yaroush. 2003. Stress and cognition: A cognitive psychological perspective. NASA/CR.

8. Heather Brown, Francesco D'Amico, Martin Knapp, et al. 2019. A cost effectiveness analysis of maintenance cognitive stimulation therapy (MCST) for people with dementia: examining the influence of cognitive ability and living arrangements. Aging \& Mental Health 23, 5: 602-607.

9. Antonio Cherubini, Carmelinda Ruggiero, Giuseppina Dell'Aquila, et al. 2012. Underrecognition and

Undertreatment of Dementia in Italian Nursing Homes.
Journal of the American Medical Directors Association 13, 8: 759.e7-759.e13.

10. Michelle Colder Carras, Antonius J. Van Rooij, Donna Spruijt-Metz, et al. 2018. Commercial Video Games As Therapy: A New Research Agenda to Unlock the Potential of a Global Pastime. Frontiers in Psychiatry 8.

11. Antoine Coutrot, Sophie Schmidt, Lena Coutrot, et al. 2019. Virtual navigation tested on a mobile app is predictive of real-world wayfinding navigation performance. PLOS ONE 14, 3: e0213272.

12. Mihaly Csikszentmihalyi. 1997. Finding flow: The psychology of engagement with everyday life. Basic Books, New York, NY, US.

13. Paul Dagum. 2018. Digital biomarkers of cognitive function. npj Digital Medicine 1, 1: 10.

14. Deirdre R. Dawson and Thomas D. Marcotte. 2017 Special issue on ecological validity and cognitive assessment. Neuropsychological Rehabilitation 27, 5: 599-602.

15. E. Ray Dorsey, Spyros Papapetropoulos, Mulin Xiong, and Karl Kieburtz. 2017. The First Frontier: Digital Biomarkers for Neurodegenerative Disorders. Digital Biomarkers.

16. Patrick Felicia, ed. 2011. Handbook of Research on Improving Learning and Motivation through Educational Games: Multidisciplinary Approaches. IGI Global.

17. Karsten Gielis, Filipa Brito, Jos Tournoy, and Vero Vanden Abeele. 2017. Can Card Games Be Used to Assess Mild Cognitive Impairment?: A Study of Klondike Solitaire and Cognitive Functions. Extended Abstracts Publication of the Annual Symposium on ComputerHuman Interaction in Play - CHI PLAY '17 Extended Abstracts, ACM Press, 269-276.

18. Karsten Gielis, Joren Kennes, Christophe De Dobbeleer, Steven Puttemans, and Vero Vanden Abeele. 2019. Collecting Digital Biomarkers on Cognitive Health Through Computer Vision and 
Gameplay: an Image Processing Toolkit for Card Games. 2019 IEEE International Conference on Healthcare Informatics (ICHI), IEEE, 127-138.

19. Karsten Gielis, Katrien Verbert, Jos Tournoy, and Vero Vanden Abeele. 2019. Age? It's in the Game: An Exploratory Study on Detection of Cognitive Aging through Card Games. 2019 CHI Play, ACM Digital Library.

20. Stuart Hagler, H. B. Jimison, and M. Pavel. 2014. Assessing Executive Function Using a Computer Game: Computational Modeling of Cognitive Processes. .

21. H. Jimison, M. Pavel, J. McKanna, and J. Pavel.

2004. Unobtrusive Monitoring of Computer Interactions to Detect Cognitive Status in Elders. IEEE Transactions on Information Technology in Biomedicine 8, 3: 248252.

22. Stephanie L. Leal, Samuel N. Lockhart, Anne Maass, Rachel K. Bell, and William J. Jagust. 2018. Subthreshold Amyloid Predicts Tau Deposition in Aging. The Journal of Neuroscience 38, 19: 4482-4489.

23. David R. Lee, John-Paul Taylor, and Alan J. Thomas. 2012. Assessment of cognitive fluctuation in dementia: a systematic review of the literature: Cognitive fluctuation in dementia: a systematic review. International Journal of Geriatric Psychiatry 27, 10: 989-998.

24. Gill Livingston, Julie Barber, Penny Rapaport, et al. 2014. START (STrAtegies for RelaTives) study: pragmatic randomised controlled trial to determine the clinical effectiveness and cost-effectiveness of a manual-based coping strategy programme in promoting the mental health of carers of people with dementia. Health Technology Assessment 18, 61: 1-242.

25. Regan Lee Mandryk and Max Valentin Birk. 2019. The Potential of Game-Based Digital Biomarkers for Modeling Mental Health. JMIR Mental Health 6, 4: e13485.
26. A.M. Mané and Emmanuel Donchin. 1989. The Space Fortress game. Acta Psychologica, 71 71, 1: 1722.

27. Misha Pavel, Holly Jimison, Stuart Hagler, and James McKanna. 2017. Using Behavior Measurement to Estimate Cognitive Function Based on Computational Models. In V.L. Patel, J.F. Arocha, and J.S. Ancker, eds., Cognitive Informatics in Health and Biomedicine: Understanding and Modeling Health Behaviors. Springer International Publishing, Cham, 137-163.

28. R. C. Petersen, R. Doody, A. Kurz, et al. 2001 Current concepts in mild cognitive impairment. Archives of Neurology 58, 12: 1985-1992.

29. Ronald C. Petersen. 2016. Mild Cognitive Impairment. Continuum: Lifelong Learning in Neurology 22, 2 Dementia: 404-418.

30. Juleen Rodakowski, Ester Saghafi, Meryl A. Butters, and Elizabeth R. Skidmore. 2015. Non-pharmacological interventions for adults with mild cognitive impairment and early stage dementia: An updated scoping review. Molecular Aspects of Medicine 43-44: 38-53.

31. Oonagh Thompson, Suzanne Barrett, Christopher Patterson, and David Craig. 2012. Examining the Neurocognitive Validity of Commercially Available, Smartphone-Based Puzzle Games. Psychology 03, 07: 525-526.

32. Victor G. Valcour, Kamal H. Masaki, J. David Curb, and Patricia Lanoie Blanchette. 2000. The Detection of Dementia in the Primary Care Setting. Archives of Internal Medicine 160, 19: 2964

33. Vero Vanden Abeele and Bob De Schutter. 2008. Meaningful Play in Elderly Life.

34. 2013. Comprehensive mental health action plan 2013-2020. World Health Organisation. 
35. 2015. World Alzheimer Report 2015: The Global Impact of Dementia. Alzheimer's Disease International.

36. 2018. The state of the art of dementia research: New frontiers. Alzheimer's Disease International, London.

37. 2019. A Dementia Strategy for Canada. Public Health Agency of Canada. 